\title{
Structure-property relationships of electroluminescent polythiophenes: role of nitrogen-based heterocycles as side chains
}

\author{
S RADHAKRISHNAN, S J ANANTHAKRISHNAN and N SOMANATHAN* \\ Polymer Laboratory, Central Leather Research Institute, Adyar, Chennai 600 020, India
}

MS received 27 April 2010; revised 28 July 2010

\begin{abstract}
A series of conjugated polythiophenes containing nitrogen-containing heterocycles as side chain, with differing substituent nature and linkage have been studied using quantum-chemical calculations. The optical properties of synthesized polymers were compared with that of model compounds with intricate structural variations. The theoretically predicted optical characteristics are correlated with the experimentally determined parameters. Experimentally determined band gap and absorption maxima found to follow the predicted trends. Single emissive layer polymeric light emitting diodes are fabricated and the structural influence on photo- and electro-emission was studied in detail. The study shows that the nature of side chain substituent such as number/position of nitrogen atoms and mode of linking of side chain plays a crucial role in deciding the geometry which in turn controls the voltage response of the electroluminescence.
\end{abstract}

Keywords. Structure-property relationship; polythiophenes; quantum-chemical calculation.

\section{Introduction}

Polymeric and oligomeric materials have shown promising properties for use in organic electronic devices such as thin-film field effect transistors (FETs), electroluminescent diodes, lasers, sensors, and photovoltaic cells (Kraft et al 1998; Skotheim et al 1998; Andersson et al 1999; Roncali 1999; Pron and Rannou 2002). Soluble organic semiconducting electronic materials have potential advantages over existing inorganic semiconductors including processability and tunable electronic properties. Among this relatively new class of materials, $\pi$-conjugated thiophene-based oligomers (nTs) and polymers (PTs) are central players because of their chemical and electrochemical stability and ready functionalization (Afzali et al 2002; Frere et al 2003; Wang et al 2004). The interest in organic electronics stems from the facile control and flexibility that organic synthetic methodology affords in functionalizing the polythiophene at the 3rd and/or 4th positions. Hence it is possible to fine-tune key optical, electronic, and electro-optical properties.

One of the current broad needs in the field is the development of $n$-type (electron transport) conjugated polymer semiconductors for improving the performance of LEDs, solar cells, and $n$-channel thin film transistors. Electron injection and transport requirements for LEDs are sufficiently different from those for photovoltaic cells and thin film transistors (Akcelrud 2003). Most of the current

\footnotetext{
*Author for correspondence (nsomanathan@rediffmail.com)
}

highly fluorescent conjugated polymers used as emissive materials in LEDs, such as the numerous arylene vinylene polymers, polyfluorenes, and also polythiophenes, are good $p$-type (hole transport) materials (Tonzola et al 2004). The use of electron-deficient heterocyclic small molecules or polymers as a separate electron transport layer or as a blend component in conjunction with the emissive material has proven very useful in improving balanced injection and recombination in organic LEDs (Thelakkat and Schmidt 1998). But these additional layers may cause inhomogenity in the device due to phase separation/crystallization which in turn affect the efficiency which lead to designing of electron transporting hole blocking groups (ETHB) incorporated single layer devices.

The present investigation involves the possibility of incorporating ETHB functional groups in 3-position of thiophene unit to achieve better efficiency and its structural influence on optical properties. The potential candidates for ETHB function are the nitrogen/oxygen containing $\pi$-electron deficient heterocyclic moieties such as oxadiazoles, triazoles, triazines, pyridines, pyrimidines, quinolines, quinoxalines and extended benzoderivatives, etc (Promislow et al 1993; Ahn et al 2000; Belmar et al 1999; Yasuda et al 2005). Hence in the present study, structure-property relationship has been focused to explore the novel polythiophene-based materials substituted with 5-membered/6-membered nitrogen heterocycles.

The inherent electronic and optical properties of these materials derive from the extended $\pi$-system. Molecular 
structure, conformation, and the degree of overlap between molecules determine the nature and magnitude of the semiconductor property like band gap. The intrinsic band gap of conjugated polymers is generally ascribed to five contributions, viz bond length alternation, aromaticity, conjugation length, substituents effects and intermolecular interactions related to the conjugated polymer backbone (Roncali et al 1987).

The band gap of poly aromatic linear $\pi$-conjugated systems (LCSs) is determined by five contributions as

$$
E_{\mathrm{g}}=E^{\delta \mathrm{r}}+E^{\theta}+E^{\mathrm{Res}}+E^{\mathrm{Sub}}+E^{\mathrm{int}},
$$

where $E^{\delta \mathrm{r}}$ is the energy related to bond length alternation (BLA); $E^{\theta}$, the mean deviation from planarity; $E^{\text {Res }}$, the aromatic resonance energy of the cycle; $E^{\text {Sub }}$, the inductive or mesomeric electronic effects of eventual substitution; and $E^{\text {int }}$ is the intermolecular or interchain coupling in the solid state.

Understanding the mutual influences of these factors will play a key role in modern molecular engineering. Quantum-mechanical calculations prove to be extremely useful when dealing with $\pi$-conjugated oligomers and polymers (Bredas 1997). Theoretical modeling of the electronic structure of conventional oligo-thiophenes has previously been performed in parallel with experimental work (Mullen and Wegner 1998). The parent oligothiophenes have been studied as polythiophene models and as interesting conjugated organic materials in their own right (Hutchison et al 2005). The utility of computational modeling has advanced with the increased accuracy of density functional methods, which can provide accurate estimates of molecular geometries, including dihedral angles and rotational barriers, dipole moments, as well as electronic structure properties such as electron affinity, ionization potential, and band gaps (Kwon and McKee 2000; Facchetti et al 2004).

The present study investigates the structure-property relationship of novel polythiophene-based materials substituted with 5-membered/6-membered nitrogen-containing heterocycles and their extended benzo derivatives. The chemical structures of the compounds are shown in figure 1. Molecular orbital calculations are applied to obtain the optimized geometries and band gaps of the thiophene oligomers. The model compounds have been synthesized and characterized using spectroscopic techniques. Theoretically obtained band gap and absorption maxima are correlated to the experimental obtained optical characteristics for solution and as thin films. The polymers are tested for their simulated end use applications in light emitting diodes.

\section{Experimental}

Among the chemical structures shown in figure 1, compounds 2, 3, 6, 7, 8, 9, 10 and 11 were chosen as model compounds and they were chemically synthesised.

\subsection{Synthesis of monomers 2, 3, 6, 7, 8 and 9}

The corresponding amines, dissolved in ethanol were reacted with thiophene-3-carboxaldehyde in the presence of catalytic amount of acetic acid. The mixture was refluxed and the resulting solid was filtered and washed with ethanol. The compound was purified by recrystallisation.

2.1a Monomer $2 \mathrm{IR}\left(\mathrm{cm}^{-1}\right)$ : 3111, 3049, 2940, 1604, $1483,1331,1169,1027,869,832,774,693,654,614 ;{ }^{1} \mathrm{H}$ NMR (300 MHz, DMSO, ppm): $8.56(s, 1 \mathrm{H}), 8.05(s, 1 \mathrm{H})$, $7.45(m, 1 \mathrm{H})$ and $7.02(m, 2 \mathrm{H}) ;{ }^{13} \mathrm{C} \mathrm{NMR}(75 \mathrm{MHz}$, DMSO, ppm) 165,154,142,140,137,128,125.

2.1b Monomer 3 IR $\left(\mathrm{cm}^{-1}\right): 3105,3046,1598,1483$, $1421,1339,1168,960,831,774,694,645,615 ;{ }^{1} \mathrm{H}$ NMR (300 MHz, DMSO, ppm): $8.26(s, 1 \mathrm{H}), 7.43(m, 1 \mathrm{H})$ and $7.07(m, 2 \mathrm{H}),{ }^{13} \mathrm{C}$ NMR (75 MHz, DMSO, ppm) 160 , $158,154,141,140,136,128,126$.

2.1c Monomer $6 \mathrm{IR}\left(\mathrm{cm}^{-1}\right): 3101,2854,1603,1558$, $1529,1489,1327,1116,1075,1046,975,863,796,653$;

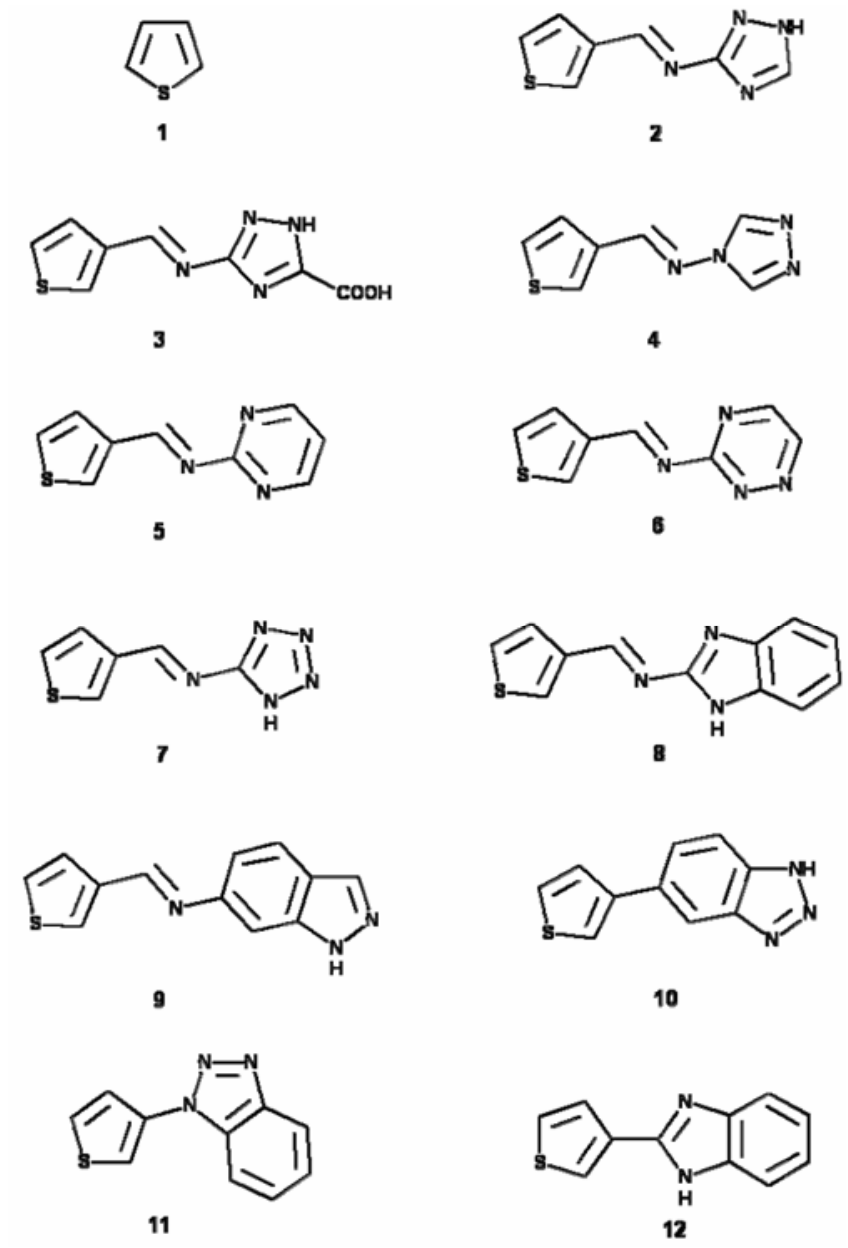

Figure 1. Different chemical structures. 
${ }^{1} \mathrm{H}$ NMR: (300 MHz, DMSO, ppm) $8 \cdot 50(d, 1 \mathrm{H}), 8 \cdot 26(s$, $1 \mathrm{H}), 8 \cdot 17(d, 1 \mathrm{H}), 7 \cdot 46(m, 1 \mathrm{H}), 7 \cdot 35(m, 2 \mathrm{H}) ;{ }^{13} \mathrm{C} \mathrm{NMR}$ (75 MHz, DMSO, ppm) 153, 149, 145, 143, 140, 137, $134,123 \cdot 5$.

2.1d Monomer 7 IR $\left(\mathrm{cm}^{-1}\right)$ : 3099, 2919, 1602, 1588, $1546,1508,1412,1235,1100,951,840,784 ;{ }^{1} \mathrm{H}$ NMR (300 MHz, DMSO, ppm): $8.25(s, 1 \mathrm{H}), 7 \cdot 51(m, 1 \mathrm{H})$, $7.45(m, 2 \mathrm{H}) ;{ }^{13} \mathrm{C}$ NMR $(75 \mathrm{MHz}, \mathrm{DMSO}, \mathrm{ppm}): 157$, $152,149,143,129,127$.

2.1e Monomer $8 \mathrm{IR}\left(\mathrm{cm}^{-1}\right): 3044,1598,1520,1495$, $1436,1401,1278,1240,1192,872,834,743,610 ;{ }^{1} \mathrm{H}$ NMR (300 MHz, DMSO, ppm): 8.28 (s, 1H), 7.69 $(d, 2 \mathrm{H}), 7.66(d, 2 \mathrm{H}), 7.46(\mathrm{~m}, 1 \mathrm{H})$ and $7.39(\mathrm{~m}, 2 \mathrm{H}) ;{ }^{13} \mathrm{C}$ NMR (75 MHz, DMSO, ppm): 160.0, 156.5, 142.9, $140 \cdot 3,135 \cdot 9,134 \cdot 8,128 \cdot 9,125 \cdot 8,122 \cdot 4,119,116,103$.

2.1f Monomer 9 IR $\left(\mathrm{cm}^{-1}\right): 3104,2967,2927,1606$, $1518,1476,1417,1360,1334,1275,1248,1204,1068$, 846, 791, 701, 654; ${ }^{1} \mathrm{H}$ NMR (300 MHz, DMSO, ppm): $8.14(s, 1 \mathrm{H}), 8.09(m, 1 \mathrm{H}), 7.98(m, 1 \mathrm{H}), 7.83(m, 1 \mathrm{H})$, $7.69(m, 1 \mathrm{H}), 7.33(m, 1 \mathrm{H})$ and $7.2(m, 2 \mathrm{H}) ;{ }^{13} \mathrm{C} \mathrm{NMR}$ (75 MHz, DMSO, ppm): 157, 156, 152.6, 149.5, $140 \cdot 2,127 \cdot 7,126 \cdot 6,125 \cdot 8,124 \cdot 6,121 \cdot 7,116 \cdot 8$ and $104 \cdot 2$.

\subsection{Synthesis of monomer $\mathbf{1 0}$}

3-bromo thiophene $(0.01 \mathrm{~mol})$ and magnesium turnings $(0.01 \mathrm{~mol})$ were introduced together with dry diethyl ether in a three-necked flask fitted with a condenser, a dropping funnel and nitrogen inlet. The entrainer 1,2dibromo ethane $(0.01 \mathrm{~mol})$ in anhydrous diethyl ether then added at ice-cold temperature for $8 \mathrm{~h}$. After the reaction started, the solution was brought to ambient temperature. The resulting Grignard compound was transferred to a second dropping funnel fitted to a second three-necked flask containing 5-chloro benzotriazole $(0.01 \mathrm{~mol})$ and 1,3-bis(diphenyl phosphino propane) nickel (II) chloride (Nidppp) in anhydrous diethyl ether. After cooling with an ice bath, the Grignard compound was added dropwise and the resulting adduct was allowed to warm up to ambient temperature and stirred for $1 \mathrm{~h}$ before being refluxed for $24 \mathrm{~h}$. The obtained mixture was poured onto very dilute aqueous hydrochloric acid. The organic layer was washed with water, dried and concentrated. The crude product was purified on a silica gel column using petroleum ether (boiling range $60-80^{\circ} \mathrm{C}$ ) as eluent.

2.2a Monomer $10 \mathrm{IR}\left(\mathrm{cm}^{-1}\right): 3280,2970,2880,2362$, $1782,1663,1606,1529,1474,1387,1322,1245,1169$, 1112, 1068, 1037, 990, 838, 737, 630; ${ }^{1} \mathrm{H} \quad \mathrm{NMR}$ (300 MHz, $\left.\mathrm{CDCl}_{3}, \mathrm{ppm}\right): 7 \cdot 29-7 \cdot 27(\mathrm{~m}, 3 \mathrm{H}), 7 \cdot 17(\mathrm{~s}$,
1H), 7.02-6.96 (m, 2H), ${ }^{13} \mathrm{C}$ NMR $(75 \mathrm{MHz}, \mathrm{DMSO}$ ppm) 139, 136, 134, 129, 128, 125, 123, 117, 115.

\subsection{Synthesis of monomer $\mathbf{1 1}$}

Monomer of compound $\mathbf{1 1}$ was prepared by microwaveassisted synthesis and the procedure followed for the synthesis is given below.

0.01 mole of $1 \mathrm{H}$-benzotriazole was dissolved in $5 \mathrm{ml}$ of chloroform. 0.01 mole of 3-bromothiophene was added to the above solution and chloroform solution was adsorbed in $20 \mathrm{~g}$ of silica gel (60-120 mesh). The silica gel was dried and subjected to microwave irradiation for $5 \mathrm{~min}$. The maximum time of reaction, microwave dosage were fixed on the basis of the study on kinetics of the reaction. The obtained product was extracted with chloroform and further treated with $1 \%$ very dilute hydrochloric acid to remove the unreacted amine. The organic layer was washed with water, dried over anhydrous sodium sulphate and purified further with column chromatography.

2.3a Monomer $11 \mathrm{IR}\left(\mathrm{cm}^{-1}\right): 2924,2854,2366,1960$, $1740,1596,1461,1212,1113,1015,774,746 ;{ }^{1} \mathrm{H}$ NMR (300 $\left.\mathrm{MHz}, \mathrm{CDCl}_{3}, \mathrm{ppm}\right): 7.96-7.94(d d, 2 \mathrm{H}), 7 \cdot 44-7.41$ $(d d, 2 \mathrm{H}), 7 \cdot 27-7.03(\mathrm{~m}, 1 \mathrm{H})$ and $7 \cdot 02-6.8(m, 2 \mathrm{H}) ;{ }^{13} \mathrm{C}$ NMR (75 MHz, $\left.\mathrm{CDCl}_{3}, \mathrm{ppm}\right): 137 \cdot 8,129 \cdot 0,128 \cdot 2,126 \cdot 9$, $126 \cdot 0,125 \cdot 3,119 \cdot 7,118 \cdot 0,117 \cdot 8$ and $115 \cdot 0$.

\subsection{Electro chemical polymerization}

The electrochemical polymerization of Schiff's bases was carried out in acetonitrile containing tetra butyl ammonium hexafluoro phosphate $\left(\mathrm{TBAPF}_{6}\right)$ as the electrolyte. Current density for the polymerization reaction was $1.43 \mathrm{~mA} / \mathrm{cm}^{2}$ and the reaction was performed for $4 \mathrm{~h}$ with the above current density. Then dedoping of the formed polymers was carried out for $30 \mathrm{~min}$ by reversing the polarity. The time of reaction and dedoping were fixed on the basis of applied current. The above procedure was adopted for synthesizing all polymers.

The absorption spectra of the samples (both in methanol solution and in thin film) were recorded using Cary UV50-Bio UV-Vis spectrophotometer. The photo emission spectrum of the sample was studied using Cary Eclipse fluorescence spectrophotometer. Thin films for the study were prepared by coating the corresponding sample solution in chloroform solvent over quartz plates using spin coating technique coated at $3000 \mathrm{rpm}$.

2.4a Fabrication and analysis of LEDs: Light emitting diodes were fabricated by spin coating the emissive polymer layer over the indium tin oxide (ITO) coated (100 nm thickness) glass plates. The thin film was formed by spin coating the chloroform solution of the polymer at $3000 \mathrm{rpm}$. Aluminum was coated over the electro emis- 
sive polymer layer, which serves as cathode and ITO acts as anode in the diode. The total emission intensity of the LEDs was tested as a function of voltage using Nucleonix Luminometer. The LEDs were tested under continuous mode of voltage application and the forward bias threshold voltage and total intensity of the emission were obtained. The wavelength of electroemission was studied using Cary Eclipse fluorescence Spectrophotometer.

\subsection{Theoretical methodology}

The Cerius ${ }^{2}$ package from Accelrys has been used for generating the initial geometries and Gaussian 98 program (Frisch et al 1998) has been used for semi-empirical AM1 and Density Functional calculation (B3LYP/6-31 $\left.\mathrm{G}^{*}\right)$. The geometries of the oligomers are optimized using Semi-empirical AM1 Hamiltonian. Then DFT (B3LYP/6$31 \mathrm{G}^{*}$ ) single point calculations have been made for geometries optimized from AM1 calculation. For comparison, the parent thiophene oligomers (1) have also been optimized.

These molecular orbital calculations are performed to estimate the band gaps $\left(E_{\mathrm{g}}\right)$ and it is calculated by taking difference between Highest Occupied Molecular Orbital (HOMO) and Lowest Unoccupied Molecular Orbital (LUMO). Band gap is extrapolated to infinite chain length by using oligomeric approach in order to get the band gap values of the polymer (Mullen and Wegner 1998). The geometry characterizations have been extended to measure the torsion angles between adjacent thiophene rings and other substituents in the side chain.

Photo absorption maxima of $\pi$-conjugated organic molecules can be predicted by ZINDO, which is parameterized explicitly for predicting excitation energies. Hence semiempirical ZINDO calculations were performed for AM1 optimized geometries. Due to limitations of the ZINDO method, structures with larger size (more number of atoms) of some of trimer and tetramer structures were not possible (Hutchison et al 2002). Absorption maxima of the polymer were obtained by plotting excitation energy against $1 / n$, where $n$ is the number of monomeric units, similar to the method mentioned above. The first excited state with significant oscillator strength was considered for the extrapolation to find the value for polymer. However, in some cases more transitions with comparable oscillator strengths are also used for comparison.

\section{Results and discussion}

Nitrogen-based heterocycles are proved to be promising candidates for the charge transporting layer in OLEDs. Substituted polythiophenes containing electron transporting group such as benzotriazole, chloro benzo triazole have been studied by Ahn et al (2001). In these studies benzotriazole was attached to the thiophene through ethyl groups, which may reduce the strain of the molecule. Hence in the present study, thiophene containing nitrogen rich hetero aromatic systems as the substituents and their benzo derivatives (fused rings) are studied systematically to understand the influence of steric and other effects of the structures on optical properties.

\subsection{Prediction of band gap}

Side chains have profound effect on band gap of electroluminescent materials (Radhakrishnan et al 2005). Electron donating (alkyl, alkoxy, alkyl amino, etc.), or withdrawing ( $\mathrm{CHO}, \mathrm{CN}, \mathrm{NO}_{2}$ etc.), substituents can raise or lower the energies of the highest occupied molecular orbital (HOMO) and the lowest unoccupied molecular orbital (LUMO) relative to the unsubstituted system, allowing rational modulation of the molecular 'band gap'. Appending electron withdrawing or donating groups onto the side chain of the polythiophene also alters the redox and optical properties. These modifications ultimately affect the charge transport characteristics of the bulk solid and define the role that the material can play in various device configurations (Facchetti et al 2004). In order to understand the role of nitrogen heterocyclic substituents on the band gap, the molecular orbital calculations are performed on oligomers and the polymer band gaps were estimated by employing oligomeric approach (Radhakrishnan et al 2005). The calculated band gap for the various structures is presented in table 1. Monomer 5 shows the maximum band gap of $5.36 \mathrm{eV}$ and 8 exhibits lowest band gap of about $3.65 \mathrm{eV}$. In absorption spectra, major blue shifts of the absorption peaks occur if large differences in the torsional angle between different rings are induced by the substituents (Hotta et al 1993a, b). The arrangement of the monomer units in substituted polythiophenes especially with bulky substituents can modify its conformational features, which in turn, govern the degree of $\pi-\pi$ conjugation between adjacent rings (Anderson et al 1995; Bolognesi et al 1999). Hence it is important to consider the torsion between the thiophene units. The schematic representation of different torsional angles is presented in figure 2. The calculated torsional angles obtained for different structures are summarized in table 2. The optimized geometry of $\mathbf{5}$ shows torsion of about $53^{\circ}(\beta)$ between segments 1 and 2 ; and about $94^{\circ}(\gamma)$ observed between segments 2 and 3 . This may reduce the $\pi$-conjugation, resulting in high band gap for the respective case. Comparison of $\mathbf{2}$ and $\mathbf{3}$ monomers show that segment 3 of monomer 2 is twisted due to the presence of electron withdrawing $\mathrm{COOH}$ group, unlike in $\mathbf{3}$, which has more planarity.

Monomers 10 and 11 are also exhibiting high band gap of 4.77 and $4.81 \mathrm{eV}$. The observed higher values of band gap in $\mathbf{1 0}$ and $\mathbf{1 1}$ can be due to the direct linkage of benzo 
triazole group linked to thiophene ring. Monomers $\mathbf{8}$ and $\mathbf{9}$ are having azomethine linkage, which can additionally contribute for extension of conjugation in molecule, leading to red shift. There is a deviation of about $38^{\circ}$ in segment $3(\gamma)$ of 9 unlike in 8 (figure 3) which has planar structure. Roncali et al (1987) and Radhakrishnan et al (2004) have reported that the steric effect of the branched alkyl chain on the polymerization behaviour is reduced when the thiophene ring is separated from branched side chain with more than two carbon atoms. Hence in the present case, introduction of $\mathrm{CH}=\mathrm{N}$ group contributes for the sterical dilution in $\mathbf{8}$ and $\mathbf{9}$ in contrast to $\mathbf{1 0}$ and $\mathbf{1 1}$ where direct linkage is involved.

The influence of the position and linkage can be better understood by comparing the band gap in 2 and 4 . The band gap value of $\mathbf{4}$ is slightly on the higher side than $\mathbf{2}$, due to the influence of the attachment of ring nitrogen to the azo methine nitrogen. Although 5 and 6 contain sixmembered rings (segment 3 ), the increase in number of nitrogen atoms and its position in the ring decreases the magnitude of band gap. The results (table 1) reveal that due to ring size, the band gap decreases. Compound 2 contains five-membered rings while $\mathbf{6}$ has six-membered rings, but contains same number of nitrogen. The variation in properties in these compounds can be attributed to the ring size.

The dimers of $\mathbf{5}$ and $\mathbf{8}$ respectively show highest and lowest band gap values. The high deviation in band gap

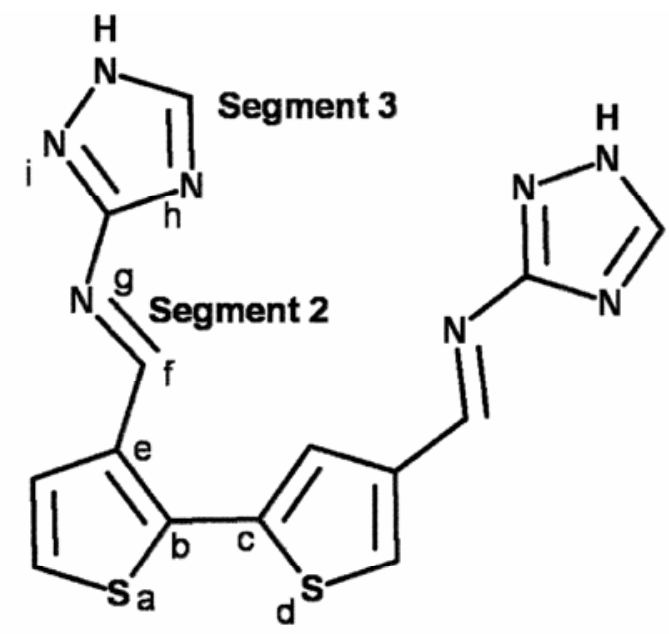

Segment 1

$$
\begin{aligned}
& \text { Inter ring torsional angle } \alpha=S_{a}-C_{b}-C_{c}-S_{d} \\
& \text { Angle } \beta=C_{e}-C_{f}-N_{g}-C_{h} \\
& \text { Angle } \gamma=C_{f}-N_{g}-C_{h}-N_{i}
\end{aligned}
$$

Figure 2. Representative figure showing different segments and angles used in this study. from thiophene in the case of $\mathbf{5}$ may be due to the torsional angle variation (table 2). The inter ring torsion angle $\alpha$, is found to be about $105^{\circ}$ for $\mathbf{5}$. In addition, the angle between segments 1 and $2(\beta)$ and segments 2 and 3 $(\gamma)$ also found to be $55^{\circ}$ and $91^{\circ}$, respectively for 5 . This resulted in the reduction of conjugation length; hence its absorptions are highly blue shifted.

Analysis of the results obtained for trimers show that 5 and 7 exhibit high and low values like in dimers. The inter ring torsion angle $(\alpha)$ of $\mathbf{5}$ is calculated to be higher than that of 7. Hence blue shift is observed in $\mathbf{5}$ compared to that of 7. Comparison of band gap of 2 and 4 show that the latter is having higher deviation value than the former. This may be attributed to the attachment of segment 2 to segment 3 through nitrogen. Monomer 7 shows lower value compared to that of $\mathbf{2}$, which may be due to the number of nitrogen atoms present in segment 3 (7).

In the case of tetramers, $\mathbf{1 0}$ shows higher band gap than 11, due to the inter ring torsion $\left(\alpha-94^{\circ}\right)$ in 10, which is higher than that of $\mathbf{1 1}$ accounting for more blue shift in 10 than in 11. Increase in planarity and reduced inter-ring torsion angle may be the reason for lowest value in $\mathbf{3}$. Comparison of band gap in tetramers of 2 and 4 shows the effect of position of linkage, viz. linkage through nitrogen, exhibiting higher deviation in 4 than 2 . The decrease in band gap in 7 compared to 2 may be due to
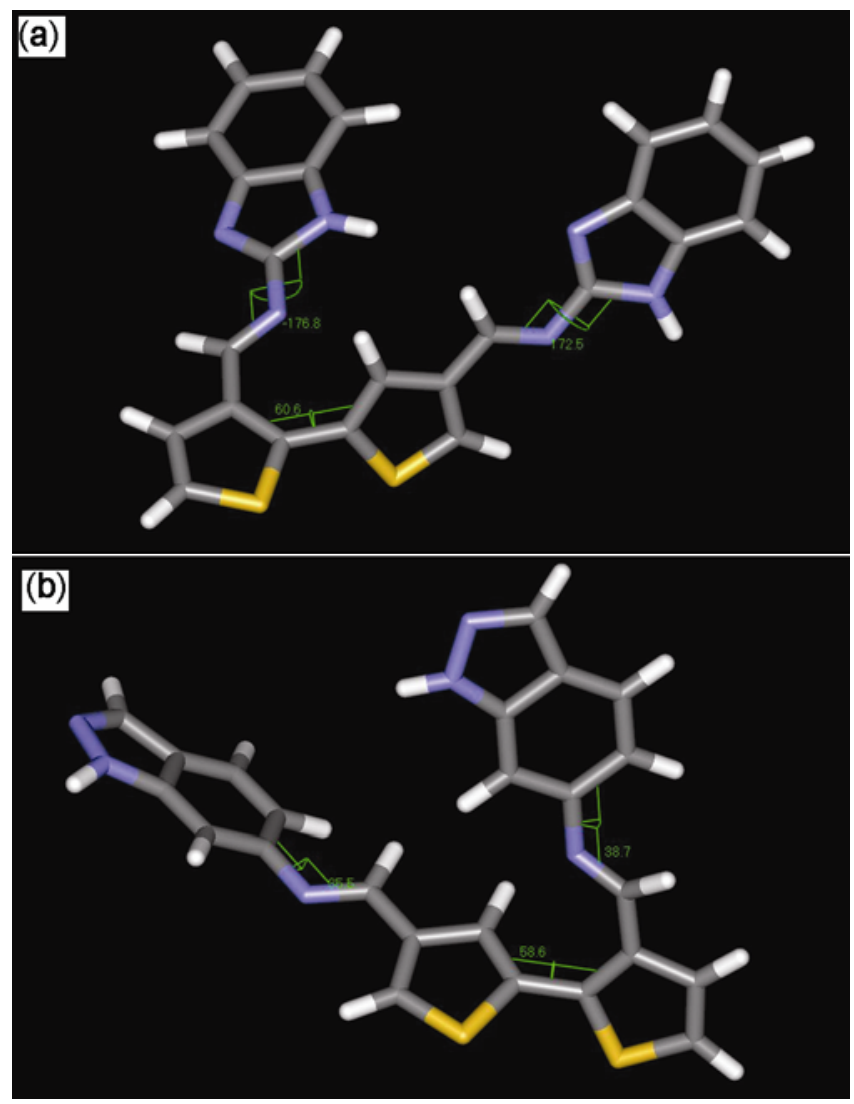

Figure 3. Optimized geometries of dimer 8 (a) and $9(\mathbf{b})$. 
Table 1. Band gap (in eV) of different compounds calculated from B3LYP/6-31G*.

\begin{tabular}{|c|c|c|c|c|c|c|c|c|}
\hline \multicolumn{2}{|c|}{ Compound } & \multirow{2}{*}{$\frac{\text { Bandgap }}{6 \cdot 391}$} & \multicolumn{2}{|c|}{ Compound } & \multirow{2}{*}{$\frac{\text { Bandgap }}{5.363}$} & \multicolumn{2}{|c|}{ Compound } & \multirow{2}{*}{$\frac{\text { Bandgap }}{4.040}$} \\
\hline 1 & $\mathrm{M}$ & & 5 & $\mathrm{M}$ & & 9 & M & \\
\hline & D & $4 \cdot 399$ & & D & $5 \cdot 024$ & & D & 3.825 \\
\hline & $\mathrm{T}$ & $3 \cdot 617$ & & $\mathrm{~T}$ & $4 \cdot 856$ & & $\mathrm{~T}$ & $3 \cdot 566$ \\
\hline & TT & $3 \cdot 214$ & & TT & $4 \cdot 543$ & & TT & $3 \cdot 555$ \\
\hline & $\mathrm{P}$ & $2 \cdot 225$ & & $\mathrm{P}$ & $4 \cdot 45$ & & $\mathrm{P}$ & $3 \cdot 401$ \\
\hline \multirow[t]{5}{*}{2} & $\mathrm{M}$ & $4 \cdot 377$ & 6 & $\mathrm{M}$ & $4 \cdot 234$ & 10 & M & 4.769 \\
\hline & D & 3.983 & & D & 4.070 & & D & $4 \cdot 731$ \\
\hline & $\mathrm{T}$ & $3 \cdot 780$ & & $\mathrm{~T}$ & $3 \cdot 604$ & & $\mathrm{~T}$ & $4 \cdot 660$ \\
\hline & TT & $3 \cdot 680$ & & TT & 3.595 & & TT & $4 \cdot 665$ \\
\hline & $\mathrm{P}$ & $3 \cdot 48$ & & $\mathrm{P}$ & $3 \cdot 424$ & & $\mathrm{P}$ & $4 \cdot 630$ \\
\hline \multirow[t]{5}{*}{3} & $\mathrm{M}$ & $4 \cdot 316$ & 7 & $\mathrm{M}$ & 4.703 & 11 & $\mathrm{M}$ & $4 \cdot 814$ \\
\hline & D & 3.996 & & D & 3.935 & & D & 4.758 \\
\hline & $\mathrm{T}$ & 3.776 & & $\mathrm{~T}$ & $3 \cdot 306$ & & $\mathrm{~T}$ & $4 \cdot 684$ \\
\hline & TT & $3 \cdot 508$ & & TT & 3.645 & & TT & $4 \cdot 579$ \\
\hline & $\mathrm{P}$ & $3 \cdot 398$ & & $\mathrm{P}$ & $3 \cdot 031$ & & $\mathrm{P}$ & 4.572 \\
\hline \multirow[t]{5}{*}{4} & $\mathrm{M}$ & $4 \cdot 394$ & 8 & $\mathrm{M}$ & $3 \cdot 649$ & 12 & $\mathrm{M}$ & 4.473 \\
\hline & D & $4 \cdot 108$ & & D & 3.491 & & D & $4 \cdot 142$ \\
\hline & $\mathrm{T}$ & 3.991 & & $\mathrm{~T}$ & $3 \cdot 385$ & & $\mathrm{~T}$ & 3.985 \\
\hline & TT & $3 \cdot 879$ & & TT & $3 \cdot 335$ & & TT & $3 \cdot 871$ \\
\hline & $\mathrm{P}$ & 3.754 & & $\mathrm{P}$ & $3 \cdot 253$ & & $\mathrm{P}$ & $3 \cdot 717$ \\
\hline
\end{tabular}

the presence of tetrazole ring (in 7) in contrast to triazole in 2.

The polymer of the structures follows the trend of tetramer structures. The magnitude of deviation increases while going from monomer to polymer. Increase in number of nitrogen atoms reduces the band gap, which can be understood, by comparing the band gap of $\mathbf{5}$ and $\mathbf{6}$, and $\mathbf{2}$ and 7. The ring size, position of linkage as well as terminal substituent may also have little influence on band gap. Analysis of the band gap shows that the band gap deviation (from unsubstituted thiophene) increases with increase in chain length.

On the basis of the results overall, the high band gap materials $(4.63-3.72 \mathrm{eV} ; \mathbf{1 0}, \mathbf{1 1}, \mathbf{5}, \mathbf{4}, \mathbf{1 2})$ are all having high torsion angle and their spatial distribution observed from its optimized geometry are also different from the geometries of the medium $(3 \cdot 48-3.40 \mathrm{eV} ; 2,6,9,3)$ and low band gap materials $(3 \cdot 25-3.00 \mathrm{eV} ; \mathbf{8}, 7)$. The optimized geometries of these strained molecules are found to distribute uniformly in space. The representative geometry of this kind is shown in figure 4 obtained for the tetramers of various structures. The side view of the geometrical structures shows that all thiophene units of tetramers fall in an axis and the side chains are evenly placed in an organized manner. This kind of 3D packing may affect the formation of supra-molecular architectures, which in turn modify the band gap. In the case of tetramers of low and mid-band gap materials, such a kind of organized geometry is not observed.

\subsection{Comparison of experimental and theoretical band gap}

The band gap of the molecules generally determined from optical absorption threshold (Yuhong et al 2000). The comparable absorption edge determined from UV-Vis spectra in solution and thin film are compared with theoretically obtained (table 3 ) band gap (overlapping absorption edge value is taken for comparison). The absorption spectra obtained for different monomers and polymers are respectively presented in figures 5 and 6 . The thin film data of monomer of some of the compounds are not presented since they did not form thin films of good optical quality. Monomer 7 is showing deviation from the theoretical order in both solution and thin film. Of all the compounds, polymer 6 shows a good overlap of experimental data with theory. It is interesting to note that except in 6, other experimentally synthesized compounds, a proton in the terminal hetero aromatic ring of the side chain is present, which it is basic. This proton may route for aggregation and polarity enhancement. It may also influence solvato chromism, which ultimately aids for deviation from theory. This view is again supported on the basis of deviations seen in polymer of $\mathbf{3}$ too, which contain polar carboxylic acid group.

The experimental trend determined from solution absorption edge of experimentally synthesized compounds follows the theoretical trend except the change in the position of 10 and 11 and only marginal change is observed in theoretical data. Monomer $\mathbf{8}$ is showing lowest band gap as predicted by theoretical calculations. Although the band gaps are showing little deviations from experimental band gaps, the trend in band gap is found to be the same in experimental and theoretical results in most of the cases. The possible reasons for the deviation in results may be (i) the gas phase is considered in the theoretical calculation, (ii) only HT-HT coupling is considered in the theoretical calculations (Belletete et al 2000). But in real case, the polymer may have regio random configura- 
Table 2. Calculated inter-ring torsional angles for the AM1 optimized geometry.

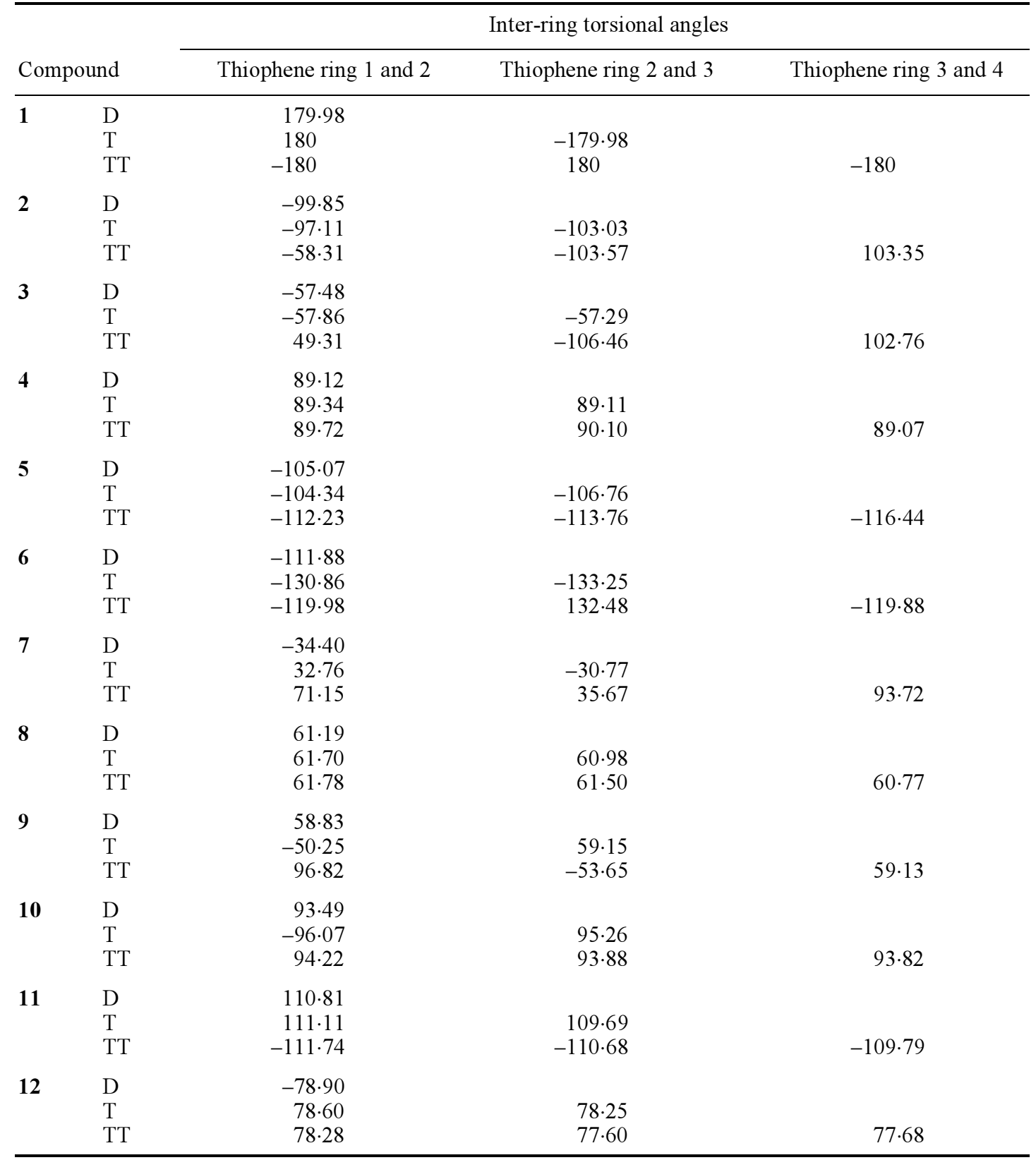

tions. (iii) Inter molecular interactions are not considered in the calculations.

\subsection{Prediction of absorption maxima and comparison with experimental results}

The absorption maxima of organic compounds can be predicted by the semiempirical molecular orbital calculations (ZINDO for electronic transition energies) (Fabian et al 1999). The peak values in the absorption spectra (solution and thin film) of monomers and polymers are determined by analysing the second derivative of the experimentally determined spectra. The predicted transi- tions from ZINDO calculations are compared with the experimentally obtained absorption maximum (values in the comparable range are considered for comparison). The transitions with maximum oscillator strength in ZINDO results are only considered for comparison with experimental results as mentioned earlier. However in some cases, oscillator strengths of more than one transition (with higher oscillator strength values) are also taken into consideration. Experimental and predicted $\lambda_{\max }$ of model compounds is presented in table 4. The results show that in general the order of $\lambda_{\max }$ observed for monomers in solution and thin film are in good agreement with the predicted trend. But in the case of $\lambda_{\max }$ 


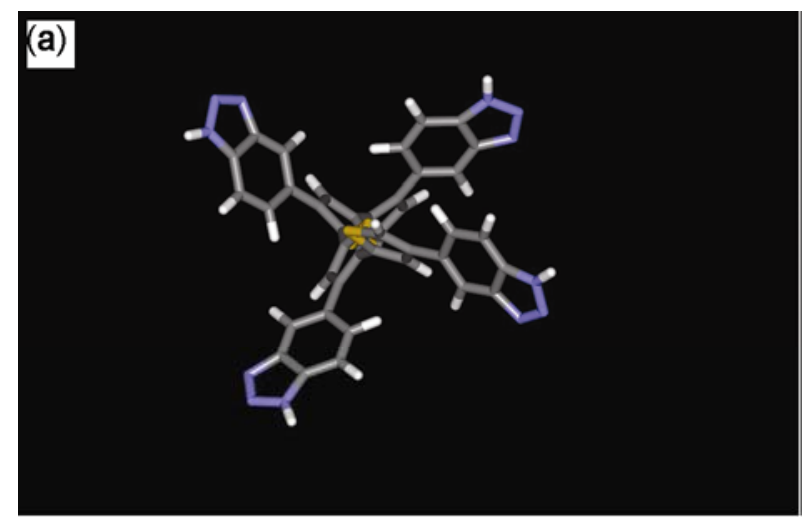

\section{(b)}
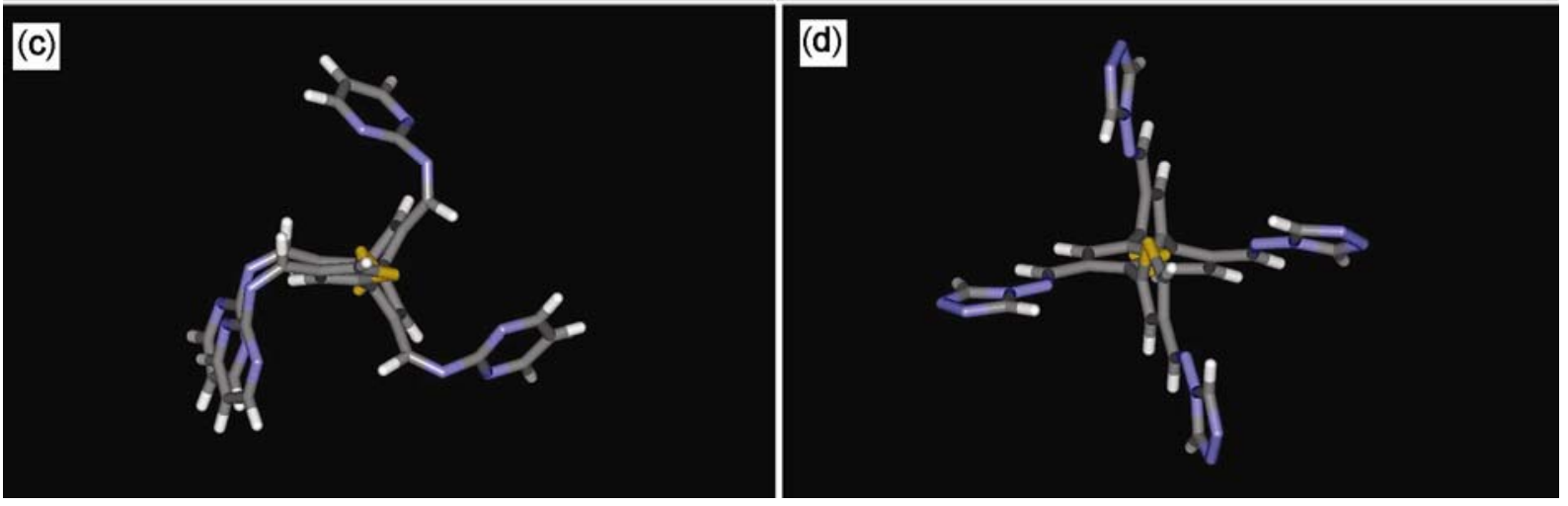

Figure 4. Optimized geometries of tetramers (side view) (contd.) compounds (a) 10; (b) 11; (c) 5; (d) 4 .

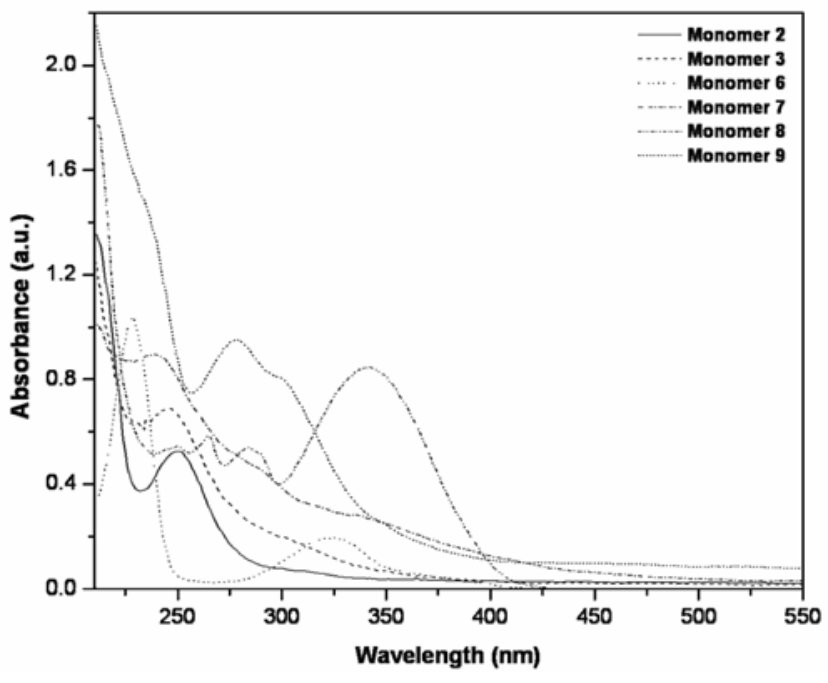

Figure 5. Absorption spectra of monomers.

obtained from polymer thin film, the reverse order of energy is observed. The energy-based ordering of compounds exactly matches with the trend obtained in band gap for the thiophene structures bearing one nitrogen heterocyclic ring at the side chain (structures 2 to 7 ). In polymeric thin films, 7 is showing high deviation in band gap, changes the order of compounds. As it was shown in the discussion with band gap earlier, the deviation in this group may be due to the protons present in triazole ring.

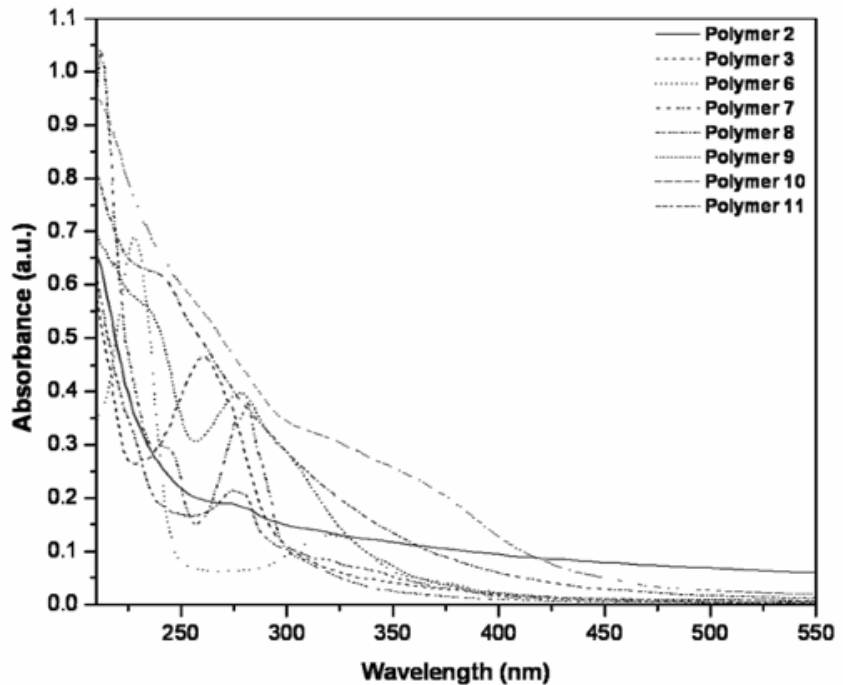

Figure 6. Absorption spectra of polymers.

In the case of 10 and 11, the predicted $\lambda_{\max }$ shows an additional peak maximum with comparable oscillator strengths $(3.81 \mathrm{eV}$ in 10 and 3.95 in 11). It is interesting to note that these additional peaks also overlap with the experimental data $(3.81 \mathrm{eV})$. The deviation in the trend of ZINDO results of polymer thin films can be attributed to the interactions involved in the polymeric chains. Even though overall ZINDO predicted $\lambda_{\max }$ is in agreement with experimental data, the slight deviation in some cases 
Table 3. Comparison of band gaps obtained by theory and experiments of monomers and polymers.

\begin{tabular}{|c|c|c|c|c|c|c|}
\hline \multirow[b]{2}{*}{ Compound no. } & \multicolumn{3}{|c|}{ Monomer } & \multicolumn{3}{|c|}{ Polymer } \\
\hline & Theo. & Solution & Thin film & Theo. & Solution & Thin film \\
\hline 2 & $4 \cdot 377$ & $4 \cdot 419$ & $4 \cdot 772$ & $3 \cdot 490$ & $4 \cdot 382$ & $4 \cdot 558$ \\
\hline 3 & $4 \cdot 316$ & $4 \cdot 192$ & $4 \cdot 661$ & 3.399 & $4 \cdot 141$ & $3 \cdot 399$ \\
\hline 6 & $4 \cdot 234$ & 3.430 & 3.941 & 3.424 & $3 \cdot 380$ & $3 \cdot 319$ \\
\hline 7 & 4.704 & 4.062 & $4 \cdot 151$ & 3.031 & 3.754 & $4 \cdot 112$ \\
\hline 8 & 3.649 & 3.078 & 3.652 & $3 \cdot 253$ & $3 \cdot 511$ & 3.909 \\
\hline 9 & 4.040 & 3.607 & 2.995 & 3.401 & 3.723 & 3.482 \\
\hline 10 & 4.769 & 4.601 & - & 4.630 & $4 \cdot 289$ & $4 \cdot 160$ \\
\hline 11 & $4 \cdot 814$ & $4 \cdot 301$ & - & 4.572 & $4 \cdot 152$ & 4.489 \\
\hline
\end{tabular}

Table 4. Comparison of absorption maxima obtained by ZINDO and experiments of monomers and polymers.

\begin{tabular}{|c|c|c|c|c|c|c|}
\hline \multirow[b]{2}{*}{ Compound no. } & \multicolumn{3}{|c|}{ Monomer } & \multicolumn{3}{|c|}{ Polymer } \\
\hline & Theo. & Solution & Thin film & Theo. & Solution & Thin film \\
\hline 2 & $4 \cdot 310$ & $4 \cdot 153$ & $4 \cdot 373$ & $3 \cdot 646$ & $3 \cdot 684$ & 3.569 \\
\hline 3 & $4 \cdot 063$ & $4 \cdot 045$ & 3.893 & $3 \cdot 046$ & $3 \cdot 007$ & 3.685 \\
\hline 6 & $3 \cdot 036$ & $3 \cdot 268$ & $3 \cdot 346$ & $3 \cdot 174$ & $3 \cdot 286$ & $3 \cdot 642$ \\
\hline 7 & $4 \cdot 845$ & $4 \cdot 721$ & $4 \cdot 889$ & $2 \cdot 227$ & $2 \cdot 878$ & $3 \cdot 924$ \\
\hline 8 & $3 \cdot 506$ & $3 \cdot 631$ & $3 \cdot 569$ & $3 \cdot 282$ & $3 \cdot 556$ & $3 \cdot 330$ \\
\hline 9 & $4 \cdot 319$ & $4 \cdot 111$ & $4 \cdot 327$ & $3 \cdot 146$ & $3 \cdot 412$ & $3 \cdot 402$ \\
\hline 10 & 4.589 & 4.633 & - & $3 \cdot 751$ & 3.707 & $3 \cdot 892$ \\
\hline 11 & 4.468 & 4.435 & - & $3 \cdot 553$ & $3 \cdot 588$ & 3.488 \\
\hline
\end{tabular}

may be due to parameterization of ZINDO. ZINDO was parameterized on small molecules and not in general on oligomers of large size. Moreover, different methods may assign different transitions as having the greatest oscillator strengths. For our purpose, the effectiveness of computational method, will be determined both by the accuracy of the resulting transition energies and by the accuracy of the predicted oscillator strengths (Hutchison et al 2002). In all the molecules consisting of greater than one monomer unit, the predicted oscillator strength of the principal transition is significantly larger than that of any other. Since our aim is to understand the influence of structure on optical properties, in this study, the other wavelength maxima obtained for different structures are also considered for discussions. The experimentally obtained absorption spectra obtained for different structures are analysed on the basis of second derivative curves and the maxima are individually compared with similar structures in the group.

\section{Influence of chemical structures on photo absorption, emission and electroemission characteristics}

\subsection{Photo absorption}

The different wavelength of absorption maxima obtained for different monomers and polymers are presented in table 5. In order to understand the effectiveness of absorption, the molar extinction coefficient of different absorptions (solutions) is also tabulated. Due to the influence of the presence of carboxyl group, the coefficient of absorption in monomer 3 increases and the influence is very much pronounced at higher wavelengths of absorption compared to monomer 2 . The attachment of the side chain groups to the main chain has a prominent influence on absorption. For example, the absorption takes higher energy and intensity (monomer 11) when the benzotriazole group is linked to thiophene through a 5 -membered ring, when compared to 10 , where the linkage of the side chain is through six-membered phenyl ring. Comparison of absorptions of 8 and 9 indicate that along with linkages, the heteroatoms present in the ring also play a role. The values obtained for monomer $\mathbf{8}$ got red-shifted than that of monomer 9. The enhancement in coefficient of absorption in $\mathbf{9}$ when compared to $\mathbf{8}$ is also observed. Similarly, due to the variation in the number of hereto atoms and their electro negativity influence, benzotriazole-containing compound (11) shows higher intensity. In polymers also, even though the main chain is the same, the absorption is modified due to the influence of side chains.

\subsection{Photoemission}

The substituents on a conjugated system may have a very profound effect on the fluorescent properties. Substituents, 
Table 5. Wavelength of absorption maxima of monomers and polymers (solution).

\begin{tabular}{|c|c|c|c|c|c|c|c|c|c|}
\hline & \multicolumn{2}{|c|}{ Monomer } & \multicolumn{2}{|c|}{ Polymer } & & \multicolumn{2}{|c|}{ Monomer } & \multicolumn{2}{|c|}{ Polymer } \\
\hline & $\lambda(\mathrm{nm})$ & $E^{\mathrm{a}, \mathrm{b}}$ & $\lambda(\mathrm{nm})$ & $E^{\mathrm{a}, \mathrm{b}}$ & & $\lambda(\mathrm{nm})$ & $E^{\mathrm{a}, \mathrm{b}}$ & $\lambda(\mathrm{nm})$ & $E^{\mathrm{a}, \mathrm{b}}$ \\
\hline \multirow[t]{14}{*}{2} & 242 & 258 & 233 & 119 & 8 & 250 & 623 & 323 & 27 \\
\hline & 249 & 292 & 243 & 95 & & 265 & 669 & 338 & 21 \\
\hline & 260 & 226 & 261 & 75 & & 283 & 614 & 372 & 10 \\
\hline & 279 & 77 & 274 & 72 & & 290 & 587 & 230 & 68 \\
\hline & 299 & 42 & 282 & 68 & & 325 & 838 & 248 & 48 \\
\hline & 309 & 37 & 297 & 58 & & 342 & 968 & 276 & 57 \\
\hline & 365 & 20 & 309 & 54 & & 365 & 708 & 283 & 64 \\
\hline & & & 337 & 47 & & & & 291 & 41 \\
\hline & & & 354 & 44 & & & & 310 & 14 \\
\hline & & & 365 & 42 & & & & 319 & 11 \\
\hline & & & 395 & 37 & 9 & 238 & 2224 & 237 & 333 \\
\hline & & & 416 & 34 & & 278 & 1533 & 241 & 307 \\
\hline & & & 430 & 32 & & 302 & 1275 & 273 & 235 \\
\hline & & & 458 & 29 & & 330 & 627 & 280 & 242 \\
\hline \multirow[t]{10}{*}{3} & 243 & 503 & 248 & 193 & & 348 & 412 & 301 & 173 \\
\hline & 248 & 502 & 253 & 220 & & & & 347 & 48 \\
\hline & 255 & 466 & 260 & 243 & & & & 364 & 29 \\
\hline & 258 & 444 & 275 & 192 & 10 & 258 & 706 & 232 & 168 \\
\hline & 261 & 421 & 300 & 58 & & 268 & 615 & 253 & 134 \\
\hline & 292 & 288 & 330 & 29 & & 284 & 478 & 264 & 122 \\
\hline & 307 & 265 & 338 & 26 & & 295 & 364 & 282 & 98 \\
\hline & 317 & 249 & 364 & 18 & & 326 & 135 & 311 & 74 \\
\hline & 349 & 213 & & & & 358 & 87 & 323 & 70 \\
\hline & 365 & 206 & & & & 381 & 72 & 335 & 65 \\
\hline \multirow[t]{8}{*}{6} & 228 & 3460 & 228 & 171 & & 400 & 60 & 348 & 60 \\
\hline & 286 & 151 & 235 & 129 & & & & 366 & 52 \\
\hline & 316 & 602 & 240 & 72 & & & & 377 & 45 \\
\hline & 330 & 623 & 245 & 33 & 11 & 251 & 800 & 230 & 33 \\
\hline & 370 & 169 & 312 & 30 & & 260 & 645 & 251 & 18 \\
\hline & 380 & 130 & 320 & 32 & & 280 & 472 & 275 & 23 \\
\hline & & & 330 & 31 & & 294 & 293 & 282 & 22 \\
\hline & & & 364 & 12 & & 326 & 81 & 306 & 11 \\
\hline \multirow[t]{7}{*}{7} & 235 & 180 & 232 & 36 & & 383 & 93 & & \\
\hline & 244 & 177 & 241 & 35 & & 402 & 98 & & \\
\hline & 263 & 128 & 244 & 35 & & & & & \\
\hline & 279 & 103 & 261 & 28 & & & & & \\
\hline & 291 & 91 & 283 & 21 & & & & & \\
\hline & 319 & 63 & 293 & 18 & & & & & \\
\hline & & & 329 & 11 & & & & & \\
\hline
\end{tabular}

${ }^{\mathrm{a}} E=$ absorbance of a $1 \%$ solution of the substance in a $1.0 \mathrm{~cm}$ cell.

${ }^{\mathrm{b}}$ Relationship between $E$ and molar extinction coefficient $10 \varepsilon=E \times$ mol. wt.

which enhance $\pi$-electron mobility, will normally increase fluorescence. A combination of electron-donating substituents with electron withdrawing substituents is used to enhance fluorescence. The present study explores the influence of different hetero aromatic functional groups on photoemission characteristics of polythiophenes. The representative photoemission of comparable structures of monomers and polymers are compared respectively in figures 7 and 8 . In order to compare all the emission wavelengths obtained from different compounds, the compounds are excited in wavelength, where coefficient of absorption was found to be high. In order to normalize different emission intensities obtained from various compounds, a normalization procedure (Radhakrishnan and Somanathan 2006) has been adopted for comparison. The maximum emission intensity obtained from a compound is taken as $100 \%$ and the other obtained intensities from photoemission experiments are normalized with reference to the above. The corresponding normalized values at different wavelengths for the synthesized monomers and polymers (solution) are presented in table 6. (The first values represent the wavelength of emission and the second values represent the normalized intensity fraction.) The experiments were performed with methanol solutions of compounds of same concentration. The thin films for emission experiments are prepared 
Table 6. Photoemission wavelengths of monomers and polymers in methanol.

\begin{tabular}{|c|c|c|c|c|c|c|c|c|c|}
\hline \multirow[b]{2}{*}{ Monome } & \multirow[t]{2}{*}{$\lambda_{\mathrm{exc}}$} & \multicolumn{8}{|c|}{ Wavelengths of emission $(\mathrm{nm}) /$ normalized intensity value } \\
\hline & & & & & & & & & \\
\hline 2 & 249 & $297 / 0.74$ & $306 / 1 \cdot 0$ & $355 / 0 \cdot 45$ & $400 / 0 \cdot 30$ & $538 / 0 \cdot 15$ & $585 / 0 \cdot 36$ & $605 / 0 \cdot 35$ & $743 / 0 \cdot 04$ \\
\hline 3 & 241 & $331 / 0 \cdot 46$ & $388 / 1 \cdot 0$ & $424 / 0 \cdot 90$ & $452 / 0 \cdot 71$ & $528 / 0 \cdot 33$ & $555 / 0.59$ & $652 / 0 \cdot 11$ & $723 / 0 \cdot 10$ \\
\hline 6 & 325 & $361 / 0 \cdot 42$ & $444 / 1 \cdot 0$ & $489 / 0 \cdot 04$ & $720 / 0 \cdot 06$ & & & & \\
\hline 7 & 240 & $308 / 0.42$ & $352 / 0.82$ & $420 / 0 \cdot 62$ & $520 / 1 \cdot 0$ & $602 / 0 \cdot 33$ & $662 / 0 \cdot 2$ & & \\
\hline 8 & 342 & $381 / 1 \cdot 0$ & $427 / 0.91$ & $463 / 0 \cdot 65$ & $616 / 0.46$ & & & & \\
\hline 9 & 278 & $373 / 1 \cdot 0$ & $460 / 0 \cdot 3$ & $724 / 0 \cdot 08$ & & & & & \\
\hline 10 & 246 & $287 / 0 \cdot 04$ & $335 / 0 \cdot 04$ & $353 / 0 \cdot 08$ & $391 / 0.96$ & $413 / 1 \cdot 0$ & $441 / 0 \cdot 51$ & & \\
\hline 11 & 281 & $366 / 0 \cdot 51$ & $432 / 1 \cdot 0$ & $679 / 0 \cdot 04$ & & & & & \\
\hline \multicolumn{10}{|c|}{ Polymer } \\
\hline 2 & 231 & $315 / 0 \cdot 85$ & $329 / 1 \cdot 0$ & $421 / 0 \cdot 18$ & $494 / 0 \cdot 11$ & $588 / 0 \cdot 20$ & $602 / 0 \cdot 23$ & $625 / 0 \cdot 26$ & $654 / 0 \cdot 22$ \\
\hline 3 & 261 & $315 / 1 \cdot 0$ & $394 / 0 \cdot 24$ & $614 / 0 \cdot 33$ & & & & & \\
\hline 6 & 229 & $318 / 0 \cdot 77$ & $328 / 1 \cdot 0$ & $338 / 0 \cdot 90$ & $439 / 0 \cdot 34$ & $490 / 0 \cdot 20$ & $583 / 0 \cdot 16$ & $625 / 0 \cdot 23$ & $648 / 0 \cdot 23$ \\
\hline 7 & 231 & $312 / 0 \cdot 56$ & $347 / 0 \cdot 91$ & $429 / 1 \cdot 0$ & $493 / 0 \cdot 88$ & $583 / 0 \cdot 42$ & $647 / 0 \cdot 23$ & $686 / 0 \cdot 17$ & \\
\hline 8 & 283 & $315 / 1 \cdot 0$ & $617 / 0 \cdot 30$ & & & & & & \\
\hline 9 & 279 & $374 / 1 \cdot 0$ & $459 / 0 \cdot 47$ & $731 / 0 \cdot 08$ & & & & & \\
\hline 10 & 262 & $327 / 0 \cdot 28$ & $412 / 0 \cdot 91$ & $430 / 1 \cdot 0$ & $457 / 0.60$ & $553 / 0 \cdot 14$ & $566 / 0 \cdot 16$ & $582 / 0 \cdot 15$ & $622 / 0 \cdot 11$ \\
\hline 11 & 276 & $305 / 1 \cdot 0$ & $321 / 0 \cdot 82$ & $598 / 0 \cdot 35$ & $586 / 0 \cdot 38$ & & & & \\
\hline
\end{tabular}

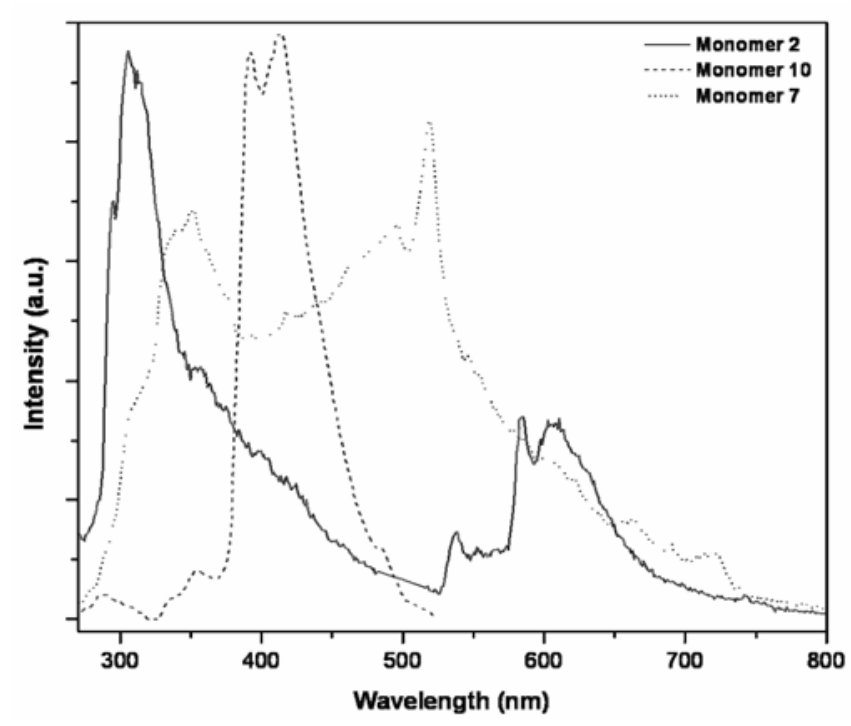

Figure 7. Photoemission spectra of monomers.

from chloroform solution. In general, the emission is obtained in all base colour ranges (blue, green and red) apart from UV range, which is one of our prime aims to get the base colours from a single thiophene molecule simultaneously. On the basis of normalized emission intensities in different wavelength regions, the prominent wavelength range of emission is fixed and the compounds in the same group are graded (ordered) on the basis of descending energy (increasing wavelength). The qualitative ordering of compounds was done by considering the wavelengths of emission and the emission intensity at different colour ranges. For example, the compound emitting more intensity in UV/blue and very low intensity at green is considered to take higher position in the emis- sion ordering of compounds. The qualitative comparison of the order of emission wavelength of model compounds in methanol solutions are

$$
\begin{array}{ll}
\text { Monomer } & \mathbf{2}>9>3>10>11>6>8>7, \\
\text { Polymer } & 11>2>6>8>3>9>7>10 .
\end{array}
$$

In most of the cases the polymers show overlapping trend with band gap order obtained from B3LYP/6-31G* calculations as well as wavelength of absorption maxima pattern obtained from ZINDO calculations. The emission pattern matches well with the order obtained from theoretical calculations. However, the order deviates from theory in some compounds, which may be due to the presence of solvation and its influence over different structures containing different functional groups.

It is observed that the structures under study have the binding on the wavelength of photoemission studied in solution. The $329 \mathrm{~nm}$ peak obtained for polymer 2 is shifted in polymer 3 to $315 \mathrm{~nm}$. Intensity of emission is also very much influenced by the structure and therefore the photoemission intensity of solutions of monomers and polymers are compared. Both monomer and polymer of 9 gave highest intensity of photoemission. Similarly, monomer and polymer of $\mathbf{1 1}$ show moderately high emission.

The photoemission is studied for different compounds, in thin films prepared from chloroform solution. Even though it is known that aggregation/packing, and therefore the optical characteristics, are influenced by the method of preparation and solvent used; chloroform was used since it gave good thin films. Some monomers do not give thin films of good optical quality.

The qualitative efficiency of thin film photoemission for the compounds is studied by adapting the procedure 
Table 7. Photoemission wavelengths of monomers and polymers in thin film.

\begin{tabular}{|c|c|c|c|c|c|c|c|c|c|}
\hline \multirow[b]{2}{*}{ Monom } & \multirow[t]{2}{*}{$\lambda_{\mathrm{exc}}$} & \multicolumn{6}{|c|}{ Wavelengths of emission (nm)/normalized intensity value } & & \\
\hline & & & & & & & & & \\
\hline 2 & 222 & $306 / 0 \cdot 58$ & $375 / 0 \cdot 65$ & $386 / 0 \cdot 74$ & $526 / 1 \cdot 0$ & $566 / 0 \cdot 96$ & $592 / 0 \cdot 49$ & & \\
\hline 3 & 240 & $317 / 0 \cdot 71$ & $393 / 0 \cdot 65$ & $428 / 1 \cdot 0$ & $552 / 0 \cdot 93$ & $598 / 0 \cdot 45$ & & & \\
\hline 6 & 230 & $311 / 0 \cdot 61$ & $367 / 0 \cdot 6$ & $531 / 1 \cdot 0$ & $563 / 0.89$ & & & & \\
\hline 7 & 238 & $324 / 0.74$ & $365 / 0 \cdot 67$ & $422 / 1 \cdot 0$ & $561 / 0.89$ & $616 / 0 \cdot 41$ & & & \\
\hline 8 & 217 & $297 / 0 \cdot 34$ & $364 / 0 \cdot 34$ & $488 / 1 \cdot 0$ & $536 / 0 \cdot 85$ & $562 / 0.99$ & $596 / 0 \cdot 40$ & $620 / 0 \cdot 24$ & $623 / 0 \cdot 26$ \\
\hline 9 & 333 & $424 / 0 \cdot 86$ & $440 / 0 \cdot 85$ & $468 / 0 \cdot 85$ & $491 / 0 \cdot 92$ & $528 / 1 \cdot 0$ & $573 / 0 \cdot 92$ & $603 / 1 \cdot 0$ & \\
\hline Polym & & & & & & & & & \\
\hline 2 & 237 & $318 / 0 \cdot 64$ & $368 / 0 \cdot 56$ & $531 / 1 \cdot 0$ & $596 / 0 \cdot 4$ & & & & \\
\hline 3 & 270 & $308 / 1 \cdot 0$ & $371 / 0.59$ & $454 / 0 \cdot 66$ & $611 / 0 \cdot 48$ & & & & \\
\hline 6 & 238 & $320 / 1 \cdot 0$ & $364 / 0 \cdot 87$ & $393 / 0 \cdot 92$ & $590 / 0 \cdot 67$ & $608 / 0 \cdot 56$ & & & \\
\hline 7 & 231 & $316 / 0.59$ & $522 / 1 \cdot 0$ & $534 / 0 \cdot 99$ & $564 / 0 \cdot 89$ & $636 / 0 \cdot 32$ & & & \\
\hline 8 & 219 & $295 / 0 \cdot 54$ & $352 / 0 \cdot 48$ & $370 / 0 \cdot 61$ & $389 / 0 \cdot 72$ & $531 / 0 \cdot 93$ & $565 / 1 \cdot 0$ & $672 / 0 \cdot 46$ & \\
\hline 9 & 218 & $303 / 0 \cdot 94$ & $320 / 0 \cdot 98$ & $362 / 0 \cdot 81$ & $398 / 1 \cdot 0$ & & & & \\
\hline 10 & 239 & $322 / 0 \cdot 56$ & $364 / 0.47$ & $425 / 0 \cdot 91$ & $534 / 1 \cdot 0$ & $550 / 0 \cdot 90$ & $639 / 0 \cdot 26$ & & \\
\hline 11 & 232 & $324 / 0 \cdot 54$ & $389 / 0.67$ & $536 / 1 \cdot 0$ & $568 / 0 \cdot 87$ & $619 / 0 \cdot 32$ & & & \\
\hline
\end{tabular}

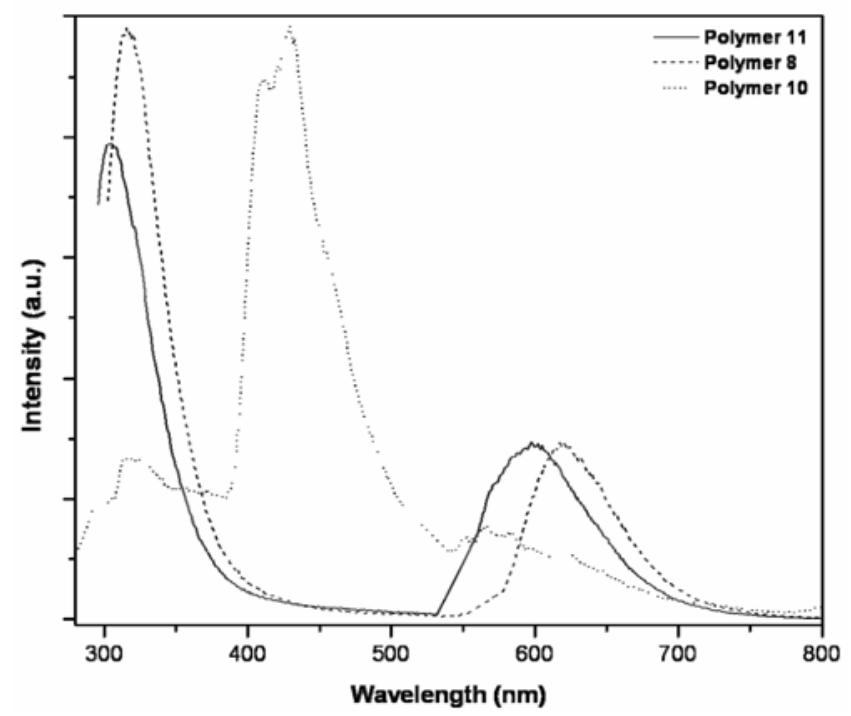

Figure 8. Photoemission spectra of polymers.

(normalization method) as mentioned earlier. In table 7 , the photoemission of the model compounds are presented and based on this, the compounds are qualitatively ordered (both monomers and polymers) by adapting the similar procedure discussed earlier. The trend obtained for thin films of the compounds of different groups is presented below.

$$
\begin{array}{ll}
\text { Monomer } & 7>3>8>2>6>9, \\
\text { Polymer } & 9>3>7>2>6>10>11>8 .
\end{array}
$$

The peaks at 308 and $352 \mathrm{~nm}$, obtained from the solution of monomer 7 are red shifted in thin films. In general, due to the interaction of solvent's polarity with compound's structure and its polarity, the packing during aggregation will be different.
The influence of different structures on the overall photoemission intensity is taken for analysis. The monomeric thin films of $\mathbf{2}$ and $\mathbf{8}$ gave the highest emission intensity while the photoemission intensity obtained for 9 is comparatively low. The photoemission intensity of polymer 8 in thin film continued to emit intense light.

\subsection{Electroemission}

Electroemission is the term used to describe the overall process that occurs, when light is emitted upon application of an electric field to a semi-conducting material. The similarity between the PL and EL spectra in luminescent materials suggest that the same emitting species is involved in each case. However, the mechanism of formations is much more complicated in the latter case. In the present study, the electroemission of single layer LEDs containing thiophene compounds as emissive layer is critically analysed in order to understand the influence of structure on electroemission intensity. The values are presented in table 8 , which represent the highest intensity values obtained in $0-5 \mathrm{~V}$ applied voltage range in continuous mode.

Indium tin oxide (ITO) and aluminum are used as anode and cathode for all the devices containing different thiophene compounds. Polymer 11 shows very high emission in comparison to other compounds; 9,2 and $\mathbf{6}$ also show moderately high emission intensities measured at the same applied voltage. The forward bias occurs in 9 polymer at $3.5 \mathrm{~V}$, which can be understood on the basis of comparatively low electron affinity values, even though electronegativity is high (table 8).

Further, the obtained total emission intensity in compounds 9,2 and 11 tremendously increases with the increase of applied voltage. Polymer 9 shows threefold increase in electroemission intensity at $5.1 \mathrm{~V}$. In polymer 
Table 8. Electroemission data obtained for various polymers.

\begin{tabular}{lcc}
\hline Compound & Intensity of emission & Threshold voltage (V) \\
\hline $\mathbf{2}$ & 4930 & $2 \cdot 9$ \\
$\mathbf{3}$ & 3680 & $3 \cdot 0$ \\
$\mathbf{6}$ & 4500 & $3 \cdot 3$ \\
$\mathbf{7}$ & 3630 & $3 \cdot 4$ \\
$\mathbf{8}$ & 1900 & $3 \cdot 0$ \\
$\mathbf{9}$ & 4060 & $3 \cdot 5$ \\
$\mathbf{1 0}$ & 2100 & $2 \cdot 8$ \\
$\mathbf{1 1}$ & 10600 & $3 \cdot 1$ \\
\hline
\end{tabular}

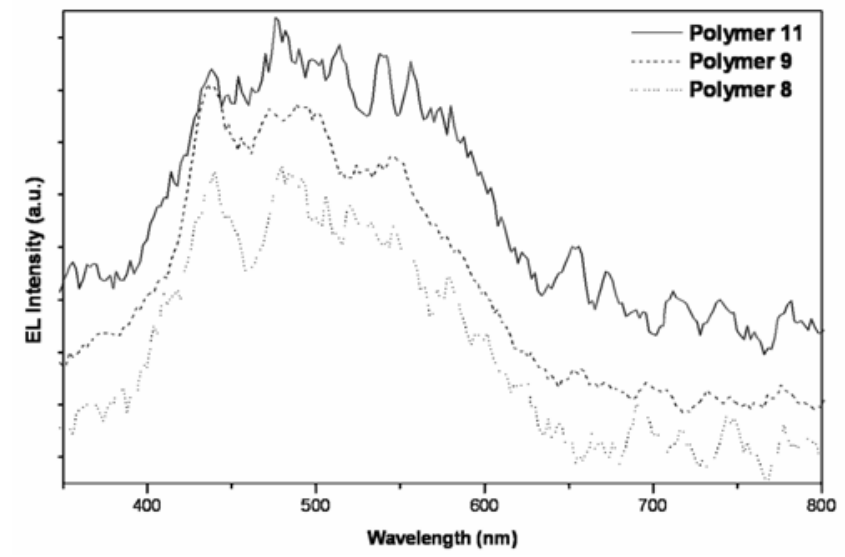

Figure 9. Electroemission of polymers.

10, the emission intensity continued to increase with the increase of voltage. A 25 -fold increase in intensity is obtained when the voltage is increased from 5 to $20 \mathrm{~V}$. Similar trend of enhancement of emission is also observed in polymers of $\mathbf{2}$ and $\mathbf{1 1}$.

Many of the other compounds taken for study do not show any characteristic change with the increase in voltage. The higher intensity of emission with voltage can be again understood on the basis of high electron affinity and high electronegativity. The overall result suggests that the electron affinity and negativity play a dominant role in enhancing electroemission intensity. The most widely used motive for electron transporting/hole-blocking functionality is based on the nitrogen containing five membered hetero aromatic rings which enhance electron affinity (Bradley et al 1998). It is interesting to note, that the results obtained from electroemission intensity with thiophene materials under study, clearly indicate that substituting appropriate hetero aromatic groups to the thiophene chain can enhance the electroemission even with single emissive layer in PLEDs.

Since it is aimed to have all base colour emissions with single emissive material, the results obtained from single layer LEDs with different polymers were analysed using spectro fluorimeter for wavelengths of electroemission, and their corresponding intensity contribution towards total emission intensity. The results obtained for model compounds are presented in table 9. The obtained electroemission spectra of some of the polymers of comparable structures are presented in figure 9. It is interesting to note that many of the polymers emit simultaneous emission in all base colours from single emissive layer LEDs.

A normalization procedure similar to photoemission normalization (explained earlier) is followed in order to understand the relation between wavelength of electroemission intensity and structure. The electroemission in the UV range could be recorded from $320 \mathrm{~nm}$ only due to the experimental limitations. This also depends on the applied voltage. Qualitative ordering of polymers on the basis of electroemission pattern (Fabian et al 1999) (on the basis of wavelength/intensity for the compounds) is shown in table 9 . The qualitative order of wavelength of electroemission overlaps with the results obtained for photoemission trend. This suggests that the excited state formation and the emitting species involved in each case may be similar.

In this study, at the applied voltage of $5 \mathrm{~V}$, the electroemission wavelength was analysed for all compounds. But the polarity of the compounds varies due to the structural contribution of the functional groups, which change the semiconducting properties and therefore the current density. Therefore, further studies at constant current density may give a true picture of overlap of photoand electroemission properties and the corresponding effect from structure. The electron density of thin films is also influenced by the method of preparation and the solvent used for spin coating, which has to be taken for consideration for further study. The output of electroemission is also dependent on factors such as charge injection ratios, charge mobilities and extra quenching processes due to electrodes and charge carriers. Therefore within the limited framework of identical conditions, the structures of the emissive layers are correlated with the electroemission intensity and the results show amazing overlap of structural influence on enhancement of electroemission in a single emissive layer containing a self-sustained electron transporting functionality. 
Table 9. Electroemission wavelengths of polymers of groups 1, 3, 4 and 5 .

\begin{tabular}{lllllllllll}
\hline Polymer & \multicolumn{7}{c}{ Wavelengths of emission (nm)/normalized intensity value } \\
\hline $\mathbf{2}$ & $358 / 0 \cdot 49$ & $410 / 0 \cdot 71$ & $437 / 0 \cdot 92$ & $473 / 1 \cdot 0$ & $501 / 0 \cdot 87$ & $530 / 0 \cdot 75$ & $561 / 0 \cdot 46$ & & \\
$\mathbf{3}$ & $398 / 0 \cdot 54$ & $418 / 0 \cdot 67$ & $436 / 0 \cdot 92$ & $442 / 0 \cdot 92$ & $487 / 1 \cdot 0$ & $511 / 0 \cdot 77$ & $538 / 0 \cdot 67$ & $572 / 0 \cdot 62$ & $590 / 0 \cdot 44$ & $618 / 0 \cdot 26$ \\
$\mathbf{6}$ & $440 / 0 \cdot 90$ & $484 / 1 \cdot 0$ & $504 / 0 \cdot 97$ & $537 / 0 \cdot 80$ & $560 / 0 \cdot 80$ & $560 / 0 \cdot 73$ & $578 / 0 \cdot 56$ & & \\
$\mathbf{7}$ & $412 / 0 \cdot 66$ & $442 / 0 \cdot 81$ & $464 / 0 \cdot 98$ & $478 / 0 \cdot 98$ & $514 / 1 \cdot 0$ & $536 / 0 \cdot 98$ & $548 / \cdot 088$ & $568 / 0 \cdot 81$ & $584 / 0 \cdot 68$ & $600 / 0 \cdot 56$ \\
$\mathbf{8}$ & $414 / 0 \cdot 53$ & $440 / 0 \cdot 97$ & $481 / 1 \cdot 0$ & $493 / 0 \cdot 97$ & $507 / 0 \cdot 88$ & $521 / 0 \cdot 84$ & $548 / 0 \cdot 78$ & $578 / 0 \cdot 59$ & $602 / 0 \cdot 38$ & $625 / 0 \cdot 2$ \\
$\mathbf{9}$ & $436 / 1 \cdot 0$ & $448 / 0 \cdot 88$ & $456 / 0 \cdot 84$ & $473 / 0 \cdot 92$ & $490 / 0 \cdot 94$ & $500 / 0 \cdot 92$ & $546 / 0 \cdot 77$ & $600 / 0 \cdot 33$ & \\
$\mathbf{1 0}$ & $436 / 0 \cdot 83$ & $476 / 0 \cdot 93$ & $501 / 1 \cdot 0$ & $519 / 0 \cdot 88$ & $542 / 0 \cdot 97$ & $563 / 0 \cdot 88$ & $576 / 0 \cdot 74$ & $589 / 0 \cdot 61$ & $603 / 0 \cdot 43$ & $615 / 0 \cdot 31$ \\
$\mathbf{1 1}$ & $438 / 0 \cdot 82$ & $476 / 1 \cdot 0$ & $514 / 0 \cdot 89$ & $556 / 0 \cdot 86$ & $580 / 0 \cdot 71$ & & & & & \\
\hline
\end{tabular}

\section{Conclusions}

Quantum chemical calculations have been performed on the polythiophenes bearing nitrogen-containing hetrocycles as the side chains. The model compounds have been synthesized and optical absorption and emission characteristics were recorded. In general, optical characteristics found to follow the theoretically predicted trend. Light emitting diodes made out of these electroluminescent polythiophenes were analysed for its wavelength of emission and intensity. Overall, this study is useful in realizing the effect of nitrogen containing heterocyclic substituents on electroluminescent properties of polythiophenes. It turns out to be clear from the study that the nature of side chain can influence the bulk and molecular properties of polymers.

\section{References}

Afzali A, Breen T L and Kagan C R 2002 Chem. Mater. 14 1742

Akcelrud L 2003 Prog. Polym. Sci. 28875

Ahn H, Czae M, Kim E R, Lee H, Ho Han H, Noh J and Hara M 2001 Macromolecules 342522

Ahn S H, Ahn T K, Han S H and Lee H 2000 Mol. Cryst \& Liq. Cryst. 349459

Anderson M R, Beggren M, Inganas O and Gustafsson G 1995 Macromolecules 287525

Andersson M R, Thomas O, Mammo W, Svensson M, Theander $\mathrm{M}$ and Inganas O 1999 J. Mater. Chem. 91933

Belletete M, Beaupre S, Bouchard J, Blondin P, Leclerc M and Durocher G 2000 J. Phys. Chem. B104 9118

Belmar J, Parra M, Zuniga C, Perez C, Munoz C, Omenat A and Serrano J L 1999 Liq. Cryst. 26389

Bolognesi A, Porzio W, Bajo G, Zannoni G and Fannig L 1999 Acta. Polym. 50151

Bradley D D C, Grell M, Grice A, Tajbakhsh A R, O'Brien D F and Bleyer A 1998 Optical Mater. 91

Bredas J L 1997 Synth. Met. 843

Fabian W M F, Niederreiter K S, Uray G and Stadlbauer W 1999 J. Molecular Struct. 477209

Facchetti A, Yoon M, Stern C L, Hutchison G R, Ratner M A and Marks T J 2004 J. Am. Chem. Soc. 12613480
Frere P, Raimundo J -M, Blanchard P, Delaunay J, Richomme P, Sauvajol J-L, Orduna J, Garin J and Roncali J 2003 J. Org. Chem. $\mathbf{6 8} 5357$

Frisch M J et al 1998 Gaussian 98W, Revision A.1; Gaussian, Inc.: Pittsburgh, PA

Hutchison G R, Ratner M A and Marks T J 2002 J. Phys. Chem. A106 10596

Hutchison G R, Ratner M A and Marks T J 2005 J. Phys. Chem. B109 3126

Hotta S and Waragai K 1993a Adv. Mater. 5896

Hotta S and Waragai K 1993b J. Phys. Chem. 977427

Kraft A, Grimsdale A C and Holmes A B 1998 Angew. Chem. Int. edn. $\mathbf{3 7} 402$

Kwon O and McKee M L 2000 J. Phys. Chem, A104 7106

Mullen K and Wegner G (eds) 1998 Electronic mater: the oligomeric approach (Wiley-VCH: Verlag $\mathrm{GmbH}$

Promislow J H, Preston J and Samulski E T 1993 Macromolecules 261793

Pron A and Rannou P 2002 Prog. Poly. Sci. 27135

Radhakrishnan S, Parthasarathi R, Subramanian V and Somanathan N 2005 J. Chem. Phys. 123164905

Radhakrishnan S and Somanathan N 2006 J. Mater. Chem. 16 2990

Radhakrishnan S, Subramanian V and Somanathan N 2004 Organic Electron. 5227

Radhakrishnan S, Subramanian V, Somanathan N, Srinivasan K S V and Ramasami T 2003 Mol. Cryst. Liq. Cryst. 390113

Ridley J and Zerner M 1973 Theor. Chim. Acta 32111

Roncali J 1997 Chem. Rev. 97173

Roncali J 1999 J. Mater. Chem. 91875

Roncali J, Garreau R, Yassar A, Marque P, Garnier F and Lemaire M 1987 J. Phys. Chem. 916706

Skotheim T A, Elsenbaumer R L and Reynolds J R 1998 Handbook of conducting polymers (New York: Marcel Dekker) 2nd edn

Thelakkat M and Schmidt H W 1998 Polym. Adv. Technol. 9 429

Tonzola C J, Alam M M, Bean B A and Jenekhe S A 2004 Macromolecules 373554

Wang J Z, Zheng Z H, Li H W, Huck W T S and Sirringhaus H 2004 Nat. Mater. 3171

Yasuda T, Imase T, Sasaki S and Yamamoto T 2005 Macromolecules 381500

Yuhong Z, Ming W, Gouxing X and Weishen Y 2000 Chemical J. Internet 217 